\title{
Long-term Remission Achieved via Combined Chemotherapy and Radiotherapy in a Non-resectable Granulocyte Colony-stimulating Factor Producing Pleomorphic Carcinoma of the Lung
}

\author{
Masaki Yamamoto ${ }^{1}$, Saki Manabe ${ }^{1}$, Yusuke Moriyama ${ }^{1}$, Hiroshi Ishii ${ }^{1}$, Shintetsu Tanaka ${ }^{1}$, \\ Ryohei Takahashi ${ }^{1}$, Koji Tomaru ${ }^{1}$, Nobuaki Kobayashi ${ }^{1}$, Makoto Kudo ${ }^{1}$, Masahiro Sasaki ${ }^{1}$, \\ Yoshiaki Inayama $^{2}$, Takeshi Kaneko ${ }^{3}$ and Yoshiaki Ishigatsubo ${ }^{4}$
}

\begin{abstract}
The prognosis is poor for patients with advanced pleomorphic carcinoma of the lung due to the generally limited response to chemotherapy and/or radiotherapy. It has been suggested the production of granulocyte colony-stimulating factor (G-CSF) by cancer cells may aggravate the disease progression. We herein report a case of a 73-year-old Japanese man with advanced G-CSF-producing pleomorphic carcinoma of the lung. First-line chemotherapy with carboplatin and paclitaxel had been suspended. Subsequent radiotherapy achieved a moderate volume reduction and an amelioration of the excessive G-CSF-related complications. Six cycles of second-line chemotherapy with docetaxel administered with good results. These combined treatments resulted in long term survival without progression of the disease.
\end{abstract}

Key words: lung cancer, pleomorphic carcinoma, granulocyte colony stimulating factor (G-CSF), chemotherapy, radiotherapy

(Intern Med 52: 2259-2263, 2013)

(DOI: 10.2169/internalmedicine.52.0701)

\section{Introduction}

Pleomorphic carcinoma of the lung is a rare subtype of lung cancer. Previous reports have described the successful management of the disease with complete surgical resection in the early stages, however, pleomorphic carcinoma of the lung has been reported to have aggressive clinical course with a poor response to both chemotherapy and radiotherapy. The prognosis is significantly worse than that of most other subsets of non-small cell lung cancer (NSCLC) (1).

Despite an intensive assessment, extraordinary leukocytosis without any infection is sometimes seen in patients with malignant tumors. Unregulated granulocyte colonystimulating factor (G-CSF) production is likely the cause of this complication. G-CSF is an inflammatory cytokine essential for the maturation of neutrophils. These unregulated G-CSF-producing tumors usually tend to progress rapidly, leading to the reportedly poor prognosis.

We herein report the case of a patient with locally advanced G-CSF producing pleomorphic carcinoma of the lung that was successfully treated with combined chemotherapy and radiotherapy.

\section{Case Report}

A 73-year-old Japanese man presented to a different hospital with abnormal shadows in the left upper lung field on chest X-ray that was taken during a routine health check in December 2009 (Fig. 1A). He had a history of smoking and

\footnotetext{
${ }^{1}$ Respiratory Medicine, Yokohama City University Hospital, Japan, ${ }^{2}$ Department of Pathology, Yokohama City University Graduate School of Medicine, Japan, ${ }^{3}$ Respiratory Disease Center, Yokohama City University Medical Center, Japan and ${ }^{4}$ Department of Internal Medicine and Clinical Immunology, Graduate School of Medicine \& Faculty of Medicine, Yokohama City University, Japan

Received for publication April 3, 2013; Accepted for publication June 2, 2013

Correspondence to Dr. Masaki Yamamoto, maskiyam@gmail.com
} 
A.

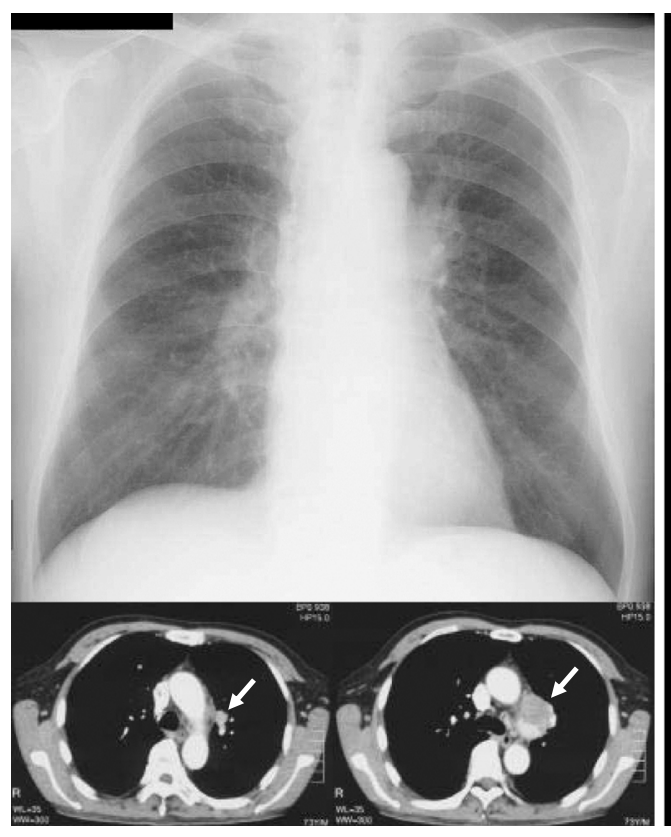

B.

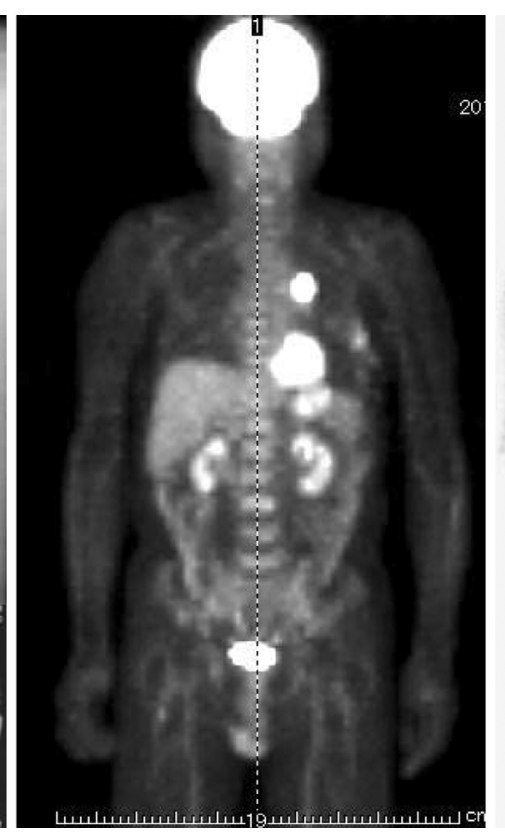

C.

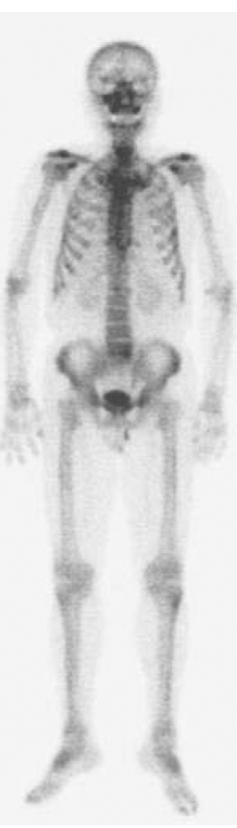

Figure 1. Diagnostic image findings. A) Chest radiograph shows an enlargement of the left hilar region of the left lung. Computed tomography (CT) scan of the chest shows a 1-cm in diameter in the left upper lobe, and a mediastinal lymphadenopathy of $3 \mathrm{~cm}$ in diameter (white arrows). B) Fluorine-18-fluorodeoxyglucose positron emission tomography (18F-FDG PET) shows increased uptake of FDG in the mediastinal lesion and in the vertebrae. The accumulation of FDG in the left chest wall matched the site of the port insertion during the video-assisted thoracoscopic surgery. C) Bone scintigraphy with technetium-99m medronic acid shows no abnormal accumulation.

had undergone surgical resection of the colon due to colon cancer at the age of 63. A doctor who had been following him after the resection noticed a slight elevation in the peripheral blood WBC and serum C-reactive protein (CRP) levels from August 2009, but chose the watch and wait approach because there were no other manifestations of any disease. Chest computed tomography (CT) revealed the presence of a nodule that was $10 \mathrm{~mm}$ in diameter and located in the left upper lobe of the lung. The nodule was attached to the massively enlarged sub-aortic and left hilar lymph nodes (Fig. 1A), suggesting a diagnosis of advanced lung cancer. Partial lobectomy to remove the lung nodule and a mediastinal lymph node biopsy were performed using video assisted thoracoscopic surgery (VATS) in January 2010. The histological findings of these specimens showed an infiltration of lymphocytes and plasmacytes in the lung as well as a few atypical cells stained with cytokeratin (CK) AE1/AE3 and CK7, and unstained with CK20, thyroid transcription factor-1 (TTF-1) and carcinoembryonic antigen (CEA) in the fibromuscular tissue. He was diagnosed with an inflammatory collapsing lesion of the lung as well as the probable lymph node metastasis of poorly differentiated carcinoma.

Fluorine-18-fluorodeoxyglucose positron emission tomography (18F-FDG PET) was performed with the aim of finding lesions that could be easily accessed by future diagnostic procedures. An abnormal accumulation of 18F-FDG was noted in the mediastinal lymph node and in the verte- brae (Fig. 1B); however, the bone scintigram revealed no abnormal accumulations (Fig. 1C). A bone marrow biopsy was performed due to bone marrow metastasis of the thoracic lesion or hematopoietic malignant disease. The biopsy samples revealed hyperplasia of the normal bone marrow without any malignant features.

The patient was referred to our hospital for further examination at the end of March 2010. He presented with a sustained fever of 38 degree Celsius as well as general fatigue. The tumor markers for lung cancer, CEA, sialyl SSEA-1 antigen (SLX), squamous cell carcinoma-related antigen (SCC), cytokeratin 19-fragment (CYFRA), neuron specific enolase (NSE) and pro-gastrin-releasing peptide (proGRP) were all within normal ranges. His peripheral blood WBC count $\left(32,700 / \mathrm{mm}^{3}\right)$ and serum CRP level $(10.8 \mathrm{mg} / \mathrm{dL}) \mathrm{had}$ increased. Culture tests showed no evidence of any bacterial infection. The mediastinal lymph node biopsy was repeated using the VATS procedure at April 2010. However, the procedure failed to reveal a definite diagnosis. Subsequently, an additional mediastinal lymph node biopsy was performed using conventional thoracotomy, through which we were finally able to retrieve an adequate specimen for pathological analysis in May 2010. A conventional histological analysis using Hematoxylin and Eosin staining showed an accumulation of spindle cells as well as both clear and foamy giant cells. Immunohistological analysis revealed positive staining for cytokeratine (AE1/AE3, CAM5.2), vimentin (2) and also G-CSF (Fig. 2). The specimens had negative result for CK7, 

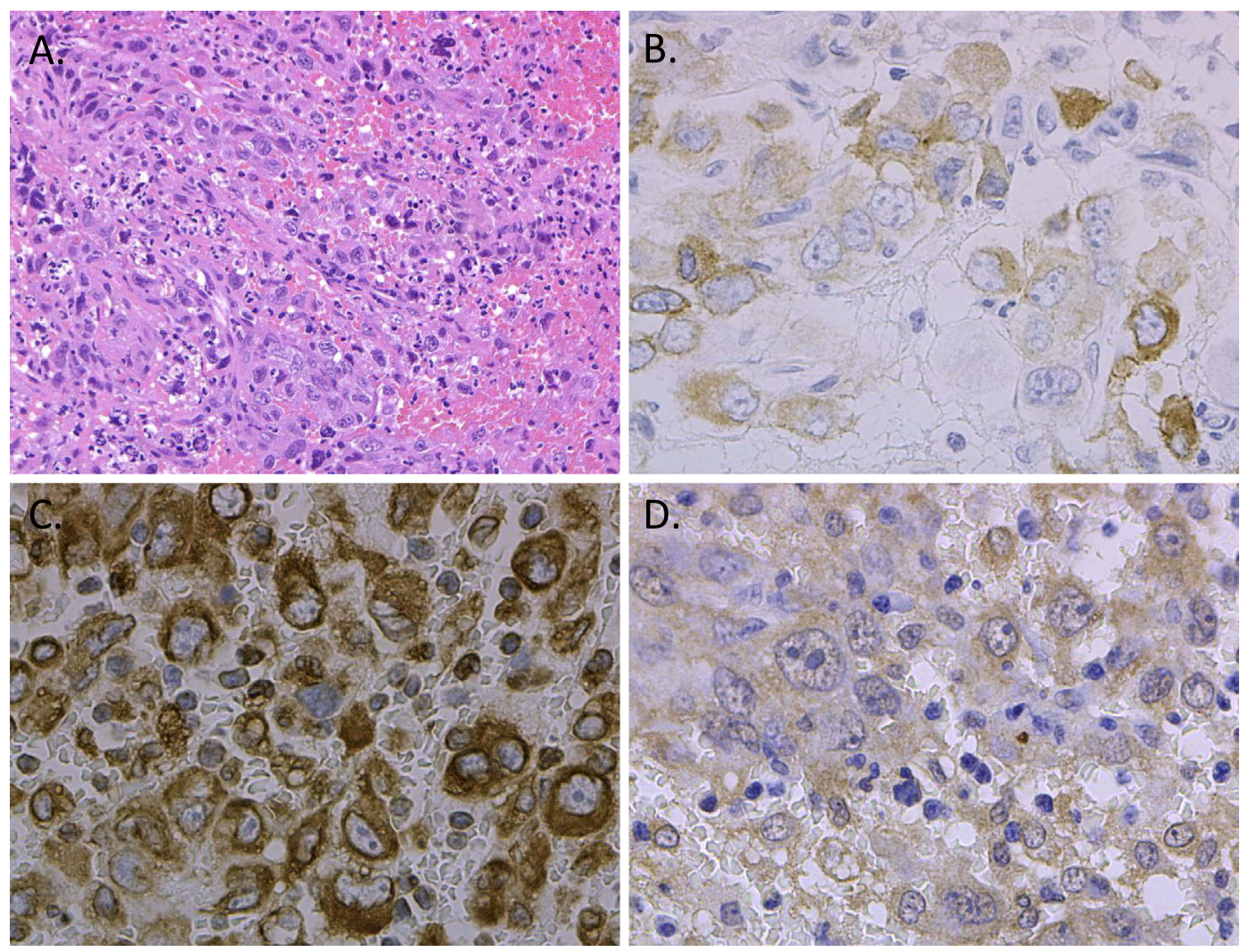

Figure 2. Histological and immunohisotochemical findings of the resected specimen. A) Giant cells and spindle cells surrounded by inflammatory cells. Hematoxylin and Eosin staining $(\times 200)$. Immunohistochemical staining for B) cytokeratin (AE1/AE3), C) vimentin and D) granulocyte colony stimulating factor $(\mathrm{G}-\mathrm{CSF})(\times \mathbf{4 0 0})$.

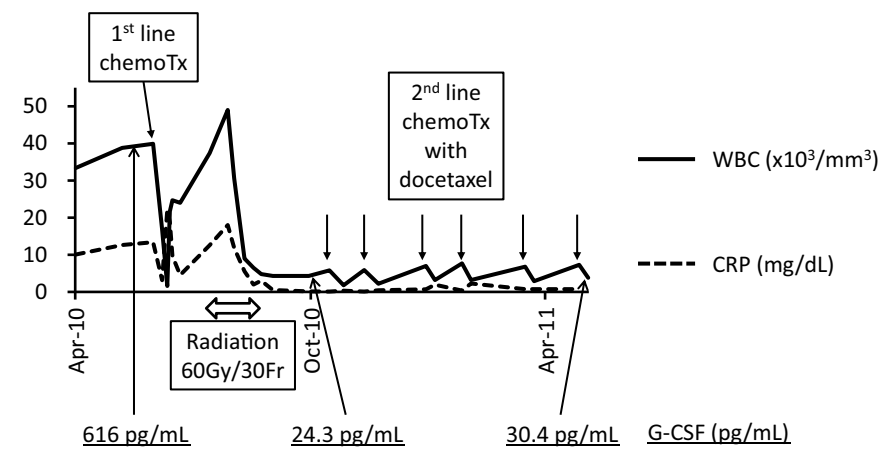

Figure 3. Clinical course of the patient during therapeutic interventions. The changes in the white blood cell count, serum CRP and G-CSF levels are shown. Horizontal axis indicates in the month and year.

CK20 and TTF-1. A diagnosis of G-CSF producing pleomorphic carcinoma was definitely confirmed. All of the complications, including the remarkable leukocytosis, the systemic inflammation and the fever, could be explained by the high serum G-CSF level (616 pg/mL; or approximately 15 times the normal upper limit). The elevated levels were likely caused by the unregulated production of G-CSF by the tumor cells. Following this initial diagnosis, the patient was completely re-evaluated, including whole body $18 \mathrm{~F}-$ FDG PET as well as brain and lumber vertebral magnetic resonance imaging. There was no evidence of metastasis to the other organs, thus suggesting a diagnosis of locally ad- vanced lung cancer (cT0N2M0, Stage IIIA). The abnormal accumulation of $18 \mathrm{~F}-\mathrm{FDG}$ in the vertebral bodies was caused by bone marrow hyperplasia due to the unregulated production of G-CSF $(3,4)$. Concurrent chemoradiotherapy was not indicated due to the age and poor performance status of the patient (PS 2).

Treatment with carboplatin $(\mathrm{AUC}=5)$ and paclitaxel $(200$ $\mathrm{mg} / \mathrm{m}^{2}$ ) was initiated as a first-line chemotherapy in mid June 2010. The combination of these drugs have shown efficacy on cancers in various organs, and a previous report described the efficacy of taxanes on in vitro drug susceptibility tests using clinically isolated pleomorphic lung carcinoma cells (1). However, evenafter initiation of the treatment, the tumor expanded from $59.9 \mathrm{~mm}$ to $83.6 \mathrm{~mm}$ on the longest axis (Fig. 4A). Moreover, these drugs caused Grade 3 anemia, leukopnea, neutropnea and platelet decreases, as well as Grade 4 arthralgia and myalgia, per the Common Terminology Criteria for Adverse Events (CTCAE), Version 4.0. Therefore, the combination of these drugs was dismissed after only a single treatment course. In order to achieve local control of the rapidly expanding mediastinal tumor, radiation therapy was chosen as an alternative treatment. A threedimensional (3D) treatment planning system was used in conjunction with $\mathrm{CT}$ in order to develop the treatment strategy. The patient was treated with a linear accelerator photon beam. The involved lesion received $60 \mathrm{~Gy}$ in conventional daily 30 fractions over six weeks. The target lesion gradu- 
A.

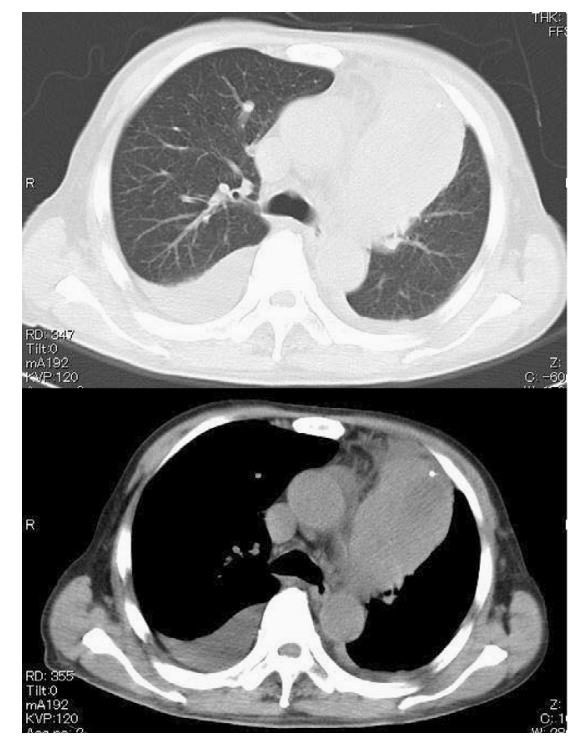

B.

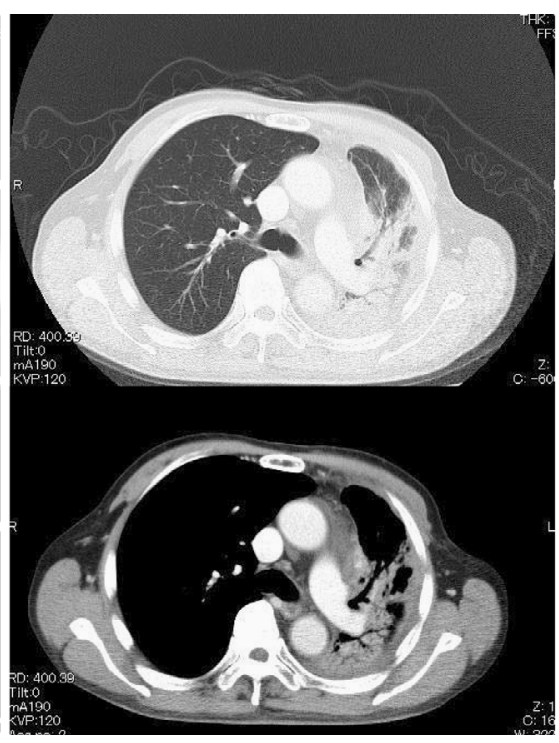

C.

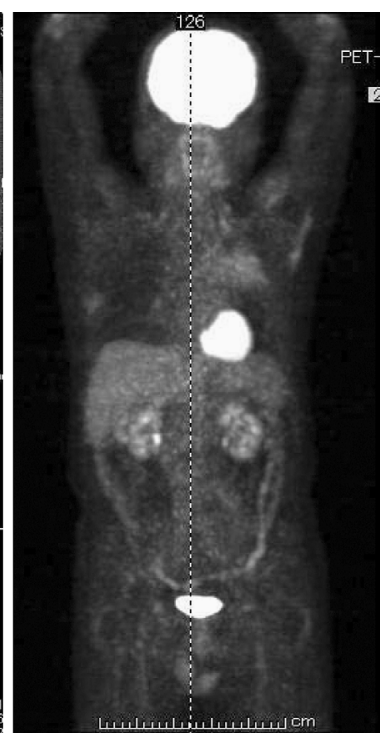

Figure 4. Image findings following therapeutic interventions. A) CT scan of the chest taken after the initiation of first-line chemotherapy shows an mediastinal lymphadenopathy of $8 \mathrm{~cm}$ in diameter as well as pleural effusion on the right. B) CT scan of the chest taken one month after 6 courses of second-line chemotherapy shows the volume reduction of the mediastinal lymphadenopathy. Radiation lung injury can also be observed in the left upper lung field. C) 18F-FDG PET at 20 months after the final course of chemotherapy shows only a slight accumulation of 18F-FDG on the radiation lung injury regions in upper lobe of the left lung.

ally reduced in size as a result of the radiation therapy. At one month after completion of radiation therapy, the longest diameter of the lesion was reduced by $18.9-\%$ (from 83.6 $\mathrm{mm}$ to $67.8 \mathrm{~mm}$ ). Remarkably, the symptoms that had been caused by the unregulated production of G-CSF steadily disappeared until they were completely ameliorated. The patient's serum G-CSF level had returned to normal by the end of radiation therapy (Fig. 3). Although the patient's general condition and performance status improved (PS 1), the expected reductions in the lesion size were likely diminishing at two months after the completion of radiation therapy. Therefore, an additional course of systemic chemotherapy was initiated. Treatment with $60 \mathrm{mg} / \mathrm{m}^{2}$ of docetaxel every 3 to 4 weeks was employed as a second-line chemotherapy and was carried out with tolerable myelosuppression (Grade 3 anemia, leukopnea, neutropnea, and platelet decreases). Although a significant reduction in the longest diameter of the lesion was not observed following two courses of the treatment (from $67.8 \mathrm{~mm}$ to $66.4 \mathrm{~mm}$ ), the reduction in the perpendicular diameter was $27.8-\%$ (from $38.6 \mathrm{~mm}$ to 27.9 $\mathrm{mm})$. In total six courses of the second-line chemotherapy were administered which resulted in $38.6 \%$ reduction in the longest diameter of the lesion [from $67.8 \mathrm{~mm}$ to $41.6 \mathrm{~mm}$ (Fig. 4B)].

At 32 months after the diagnosis (20 months after the final administration of chemotherapy), PET-CT revealed no growth and/or accumulation of FDG on either the treated lesion or any other sites (Fig. 4C). The serum G-CSF levels, WBC counts and serum CRP levels have also been within the normal ranges. The patient still has remained in good health with only mild complications due to radiation lung injury, including a cough and shortness of breath with moderate to difficult exercise.

\section{Discussion}

Pleomorphic carcinoma of lung was first classified in 1999 by the World Health Organization as a subset of sarcomatoid carcinoma. This type of tumor is extremely rare, accounting for only 0.3 to $1.3 \%$ of all previously reported lung malignancies $(2,5-7)$. The tumor is defined as a carcinoma that contains either components of spindle and giant cells alone, or at least $10 \%$ of spindle and/or giant cells with other typical NSCLC components such as adenocarcinoma, squamous cell carcinoma or large cell carcinoma. Because it is difficult to identify all of these features from small specimens, the diagnosis of this tumor is usually made from surgically resected specimens. In this case, only spindle shape cells were seen in specimens taken at the first biopsy. These cells were initially considered to be fibroblasts in the fibrotic tissue that surrounded the cancer cells. Eventually, specimens were obtained during thoracotomy that contained both spindle and giant cells. These cells were positively stained for both epithelial and stromal marker, cytokeratin and vimentin, respectively, therefore leading to the diagnosis of pleomorphic carcinoma.

G-CSF production has been reported in cancers of various organs. The diagnostic criteria for G-CSF producing tumors are as follows: 1) extreme leukocytosis, 2) elevated G-CSF activity, 3) decreased WBC count after tumor resection, and 
4) proof of G-CSF production in the tumor (8). In the present case, although the WBC count was normalized following radiation therapy instead of surgical resection, all of these criteria were fulfilled.

Pleomorphic carcinomas tend to grow rapidly and invade surrounding organs (1). Furthermore, metastatic lesions are likely to be seen immediately after surgical resection (9) or at the time of diagnosis. These aggressive features and the limited efficacy of chemotherapy and/or radiotherapy make the prognosis of this tumor type poor. In addition, G-CSF production by cancer cells has been associated with the rapid progression of the disease and with the poor prognosis. Although the mechanism of the G-CSF-related acceleration of cancer progression has not yet been elucidated, a direct effect on the proliferation of non-hematopoietic cancer cells $(10,11)$, and an immune-modulatory and angiogenic effect on the immune cells and the stromal cells $(12,13)$ have both been proposed. Our patient did not exhibit metastatic lesions in any other organs; however, a case of locally advanced non-resectable pleomorphic carcinoma with GCSF production was expected to show a poor prognosis. Not surprisingly, the first-line chemotherapy of carboplatin and paclitaxcel completely failed to show any efficacy for either the tumor growth or any related symptoms. Fortunately, the subsequent radiation therapy successfully achieved a reduction in the tumor size as well as a decrease G-CSF production. Similar benefits of radiation therapy for G-CSF producing tumors has been reported previously (14). The reduction of in the production of G-CSF led to the remission of the clinical manifestations, including fever and general fatigue. The radiotherapy improved patient's general condition allowed us to subsequently initiate chemotherapy with docetaxel. The potency of this agent had been demonstrated previously through an in vitro drug susceptibility test with surgically resected tissues as well as a clinical case report (1). Our patient also showed a favorable response to the chemotherapy agent.

Since this subtype of lung cancer is rare, there have been very few case reports or retrospective analysis concerning the treatment of advanced pleomorphic carcinoma. Recently, characterizations of the mutational or amplification status of several well-known oncogenic target genes in this type of cancer have been reported $(15,16)$. It is expected that clinical trials based on this cancer-genotyping will be conducted as an alternative treatment for this rare cancer in the future.

In summary, we successfully controlled a locally advanced G-CSF producing pleomorphic carcinoma of lung. The amelioration of the systemic manifestation caused by the unregulated production of G-CSF using radiation therapy allowed us to initiate potent chemotherapy, and the combination of the two therapies provided a favorable outcome. As previously reported (1), docetaxel showed great potency for the treatment of pleomorphic carcinoma. We demon- strated that the reduction of G-CSF production in G-CSF producing tumors plays important roles in improving the patient's general condition and maintaining continuity of further treatments.

The authors state that they have no Conflict of Interest (COI).

\section{References}

1. Kawano R, Hino H, Hoshino T, et al. Clinicopathological investigation of seven resected cases of pleomorphic carcinoma of the lung, and the results of their chemosensitivity tests. Haigan (Jpn J Lung Cancer) 48: 106-111, 2008 (in Japanese, Abstract in English).

2. Terada T. Pleomorphic carcinoma of the lung: a case report with immunohistochemical studies. Respiratory Medicine CME 3: 252256, 2010.

3. Knopp MV, Bischoff H, Rimac A, Oberdorfer F, vanKaick G. Bone marrow uptake of fluorine-18-fluorodeoxyglucose following treatment with hematopoietic growth factors: initial evaluation. Nucl Med Biol 23: 845-849, 1996.

4. Morooka M, Kubota K, Murata Y, et al. ${ }^{18}$ F-FDG-PET/CT findings of granulocyte colony stimulating factor (G-CSF)-producing lung tumors. Ann Nucl Med 22: 635-639, 2008.

5. Fishback NF, Travis WD, Moran CA, Guinee DG Jr, McCarthy WF, Koss MN. Pleomorphic (spindle/giant cell) carcinoma of the lung. A clinicopathologic correlation of 78 cases. Cancer 73: 2936-2945, 1994.

6. Nakajima M, Kasai T, Hashimoto H, Iwata Y, Manabe H. Sarcomatoid carcinoma of the lung: a clinicopathologic study of 37 cases. Cancer 86: 608-616, 1999.

7. Rossi G, Cavazza A, Sturm N, et al. Pulmonary carcinomas with pleomorphic, sarcomatoid, or sarcomatous elements: a clinicopathologic and immunohistochemical study of 75 cases. Am J Surg Pathol 27: 311-324, 2003.

8. Asano S, Urabe A, Okabe T, Sato N, Kondo Y. Demonstration of granulopoietic factor(s) in the plasma of nude mice transplanted with a human lung cancer and in the tumor tissue. Blood 49: 845$852,1977$.

9. Yuki T, Sakuma T, Ohbayashi C, et al. Pleomorphic carcinoma of the lung: a surgical outcome. J Thorac Cardiovasc Surg 134: 399404, 2007.

10. Thacker JD, Dedhar S, Hogge DE. The effect of GM-CSF and GCSF on the growth of human osteosarcoma cells in vitro and in vivo. Int J Cancer 56: 236-243, 1994.

11. Pei XH, Nakanishi Y, Takayama K, Bai F, Hara N. Granulocyte, granulocyte-macrophage, and macrophage colony-stimulating factors can stimulate the invasive capacity of human lung cancer cells. Br J Cancer 79: 40-46, 1999.

12. Natori T, Sata M, Washida M, Hirata Y, Nagai R, Makuuchi M. G-CSF stimulates angiogenesis and promotes tumor growth: potential contribution of bone marrow-derived endothelial progenitor cells. Biochem Biophys Res Commun 297: 1058-1061, 2002.

13. Okazaki T, Ebihara S, Asada M, Kanda A, Sasaki H, Yamaya M. Granulocyte colony-stimulating factor promotes tumor angiogenesis via increasing circulating endothelial progenitor cells and Gr1+ CD11b+ cells in cancer animal models. Int Immunol 18: 1-9, 2006.

14. Toyoda M, Chikamatsu K, Sakakura K, et al. A case of squamous cell carcinoma of the head and neck producing granulocyte-colony stimulating factor with marked leukocytosis. Auris Nasus Larynx 34: 267-271, 2007.

15. Chang YL, Wu CT, Shih JY, Lee YC. EGFR and p53 status of pulmonary pleomorphic carcinoma: implications for EGFR tyrosine kinase inhibitors therapy of an aggressive lung malignancy. Ann Surg Oncol 18: 2952-2960, 2011.

16. Lee S, Kim Y, Sun JM, et al. Molecular profiles of EGFR, K-ras, c-met, and FGFR in pulmonary pleomorphic carcinoma, a rare lung malignancy. J Cancer Res Clin Oncol 137: 1203-1211, 2011.

(C) 2013 The Japanese Society of Internal Medicine http://www.naika.or.jp/imonline/index.html 